\title{
Monoclonal antibodies for the S2 subunit of spike of SARS-CoV-1 cross-react with the newly-emerged SARS- CoV-2
}

Zhiqiang Zheng ${ }^{1,2}$, Vanessa Marthe Monteil3,4, Sebastian Maurer-Stroh ${ }^{5,6,7}$, Chow Wenn Yew ${ }^{8}$, Carol Leong ${ }^{8}$, Nur Khairiah MohdIsmail ${ }^{1,2}$, Suganya Cheyyatraivendran Arularasu ${ }^{1,2}$, Vincent Tak Kwong Chow ${ }^{1}$, Raymond Tzer Pin Lin ${ }^{7,9}$, Ali Mirazimi3,4,10 , Wanjin Hong $^{8}$, Yee-J00 Tan ${ }^{1,2,8}$

1. Infectious Diseases programme, Department of Microbiology and Immunology, Yong Loo Lin School of Medicine, National University Health System (NUHS), National University of Singapore, Singapore

2. Immunology programme, Department of Microbiology and Immunology, Yong Loo Lin School of Medicine, National University Health System (NUHS), National University of Singapore, Singapore

3. Department of Laboratory Medicine, Karolinska Institute, Huddinge, Sweden

4. Public Health Agency of Sweden, Stockholm, Sweden

5. Bioinformatics Institute (BII), A*STAR (Agency for Science, Technology and Research), Singapore

6. Department of Biological Sciences (DBS), National University of Singapore, Singapore

7. National Public Health Laboratory (NPHL), National Centre for Infectious Diseases (NCID), Singapore

8. Institute of Molecular and Cell Biology (IMCB), A*STAR (Agency for Science, Technology and Research), Singapore

9. Department of Microbiology and Immunology, Yong Loo Lin School of Medicine, National University Health System (NUHS), National University of Singapore, Singapore

10. National Veterinary Institute, Uppsala, Sweden

Correspondence: Yee-Joo Tan (yee_joo_tan@nuhs.edu.sg)

Citation style for this article:

Zheng Zhiqiang ORCID icon, Monteil Vanessa Marthe, Maurer-Stroh Sebastian, Yew Chow Wenn , Leong Carol, Mohd-Ismail Nur Khairiah, Cheyyatraivendran

Arularasu Suganya, Chow Vincent Tak Kwong, Lin Raymond Tzer Pin, Mirazimi Ali, Hong Wanjin, Tan Yee-Joo. Monoclonal antibodies for the S2 subunit of spike of

SARS-CoV-1 cross-react with the newly-emerged SARS-CoV-2. Euro Surveill. 2020;25(28):pii=2000291. https://doi.org/10.2807/1560-7917.ES.2020.25.28.2000291

Article submitted on 12 Mar 2020 / accepted on 11 Jun 2020 / published on 16 Jul 2020

Background: A novel coronavirus, SARS-CoV-2, which emerged at the end of 2019 and causes COVID-19, has resulted in worldwide human infections. While genetically distinct, SARS-CoV-1, the aetiological agent responsible for an outbreak of severe acute respiratory syndrome (SARS) in 2002-2003, utilises the same host cell receptor as SARS-CoV-2 for entry: angiotensin-converting enzyme 2 (ACE2). Parts of the SARSCoV-1 spike glycoprotein (S protein), which interacts with ACE2, appear conserved in SARS-CoV-2. Aim: The cross-reactivity with SARS-CoV-2 of monoclonal antibodies (mAbs) previously generated against the S protein of SARS-CoV-1 was assessed. Methods: The SARS-CoV-2 S protein sequence was aligned to those of SARS-CoV-1, Middle East respiratory syndrome (MERS) and common-cold coronaviruses. Abilities of mAbs generated against SARS-CoV-1 S protein to bind SARS-CoV-2 or its S protein were tested with SARSCoV-2 infected cells as well as cells expressing either the full length protein or a fragment of its S2 subunit. Quantitative ELISA was also performed to compare binding of mAbs to recombinant $S$ protein. Results: An immunogenic domain in the S2 subunit of SARS-CoV-1 $S$ protein is highly conserved in SARS-CoV-2 but not in MERS and human common-cold coronaviruses. Four murine $\mathrm{mAbs}$ raised against this immunogenic fragment could recognise SARS-CoV-2 S protein expressed in mammalian cell lines. In particular, mAb $1 A 9$ was demonstrated to detect $S$ protein in SARS-CoV-2infected cells and is suitable for use in a sandwich
ELISA format. Conclusion: The cross-reactive mAbs may serve as useful tools for SARS-CoV-2 research and for the development of diagnostic assays for COVID-19.

\section{Introduction}

The severe acute respiratory syndrome coronavirus (SARS-CoV-1), a virus considered to have a zoonotic origin, is the aetiological agent for the infectious disease, SARS, which first emerged in 2002-2003 [1,2]. In December of 2019, another novel coronavirus (SARSCoV-2), which causes coronavirus disease (COVID-19), appeared to have crossed species barriers to infect humans and was effectively transmitted from person to person, leading to an outbreak in Wuhan, China [3-5]. This virus subsequently spread worldwide, leading the World Health Organization (WHO) to declare a pandemic on 11 March 2020 [6]. To date, SARS-CoV-2 continues to pose a high global health and economy burden, and as at 3 May 2020, COVID-19 had affected 215 countries with over 3.35 million confirmed cases. To tackle the problems caused by SARS-CoV-2, improving its detection and knowledge of its infection mechanism is important. In this respect, the viral surface spike glycoprotein (S protein) has been demonstrated to play key role in host cell selectivity and binding. The $S$ protein is functionally divided into two subunits, with the $\mathrm{S}_{1}$ subunit containing the receptor binding domain (RBD), which allows attachment to host cells, and the 
Multiple sequence alignment for the S2 subunit fragment of SARS-CoV-1 spike glycoprotein with other relevant coronaviruses

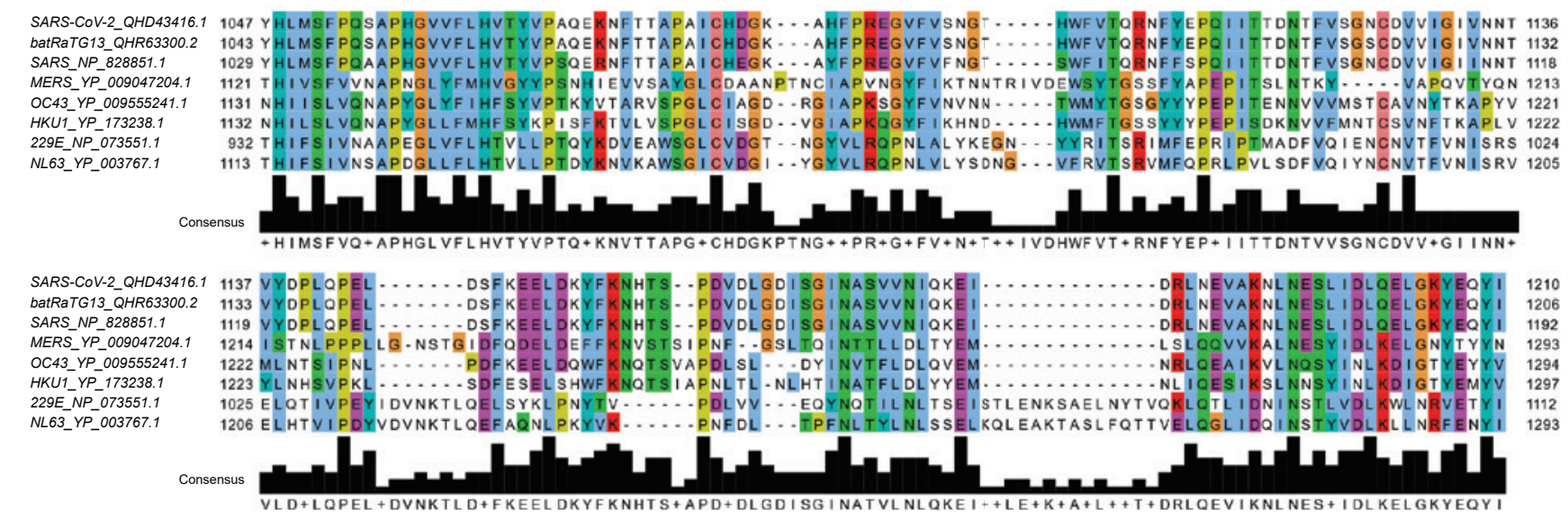

MERS: Middle East respiratory syndrome; SARS: severe acute respiratory coronavirus 1; SARS-CoV-2: severe acute respiratory coronavirus 2.

The name of the viruses, for which sequences are being compared figure on the left side of the alignment, together with the respective sequences' GenBank accession numbers.

Colour schemes represent the following categories of amino acids: blue - hydrophobic, cyan - aromatic, green - polar, magenta - negative charge, orange - glycines, pink - cysteines, red - positive charge, yellow - prolines, white - unconserved.

S2 subunit mediating fusion between viral and host membranes (reviewed by Li, F.) [7].

Phylogenetic analysis revealed that like SARS-CoV-1 and bat-derived SARS-like coronaviruses (SL-CoVs), SARS-CoV-2 belongs to lineage $B$ of the betacoronavirus genus $[8,9]$. A study of 56 complete and partial SARS-CoV-2 genomes isolated from COVID-19 patients showed very high sequence conservation of more than $99 \%$, indicating a recent introduction of the virus into the human population [10]. Although the animal source of SARS-CoV-2 is not clear, SARS-CoV-1 is believed to have originated from SL-CoVs residing in bats [11-14]. For the majority of SL-CoVs, the $\mathrm{S}_{1}$ subunit has low sequence identity to that of SARS-CoV-1, which suggests species-dependent receptor binding $[14,15]$. On the other hand, the high amino acid sequence identity of more than $90 \%$ in the S2 subunit suggests that the fusion mechanism during virus infection is well-conserved $[14,15]$.

While SARS-CoV-2 shares higher whole-genome sequence identity with bat-SL-CoVZC 45 and bat-SL-CoVZXC21 (88-89\%) than with SARS-CoV-1 (79-82\%), the RBD of SARS-COV-2 is more similar to SARS-CoV-1 RBD $[8,9]$. In line with this, several research groups have demonstrated that SARS-CoV-2 utilises the same host receptor, angiotensin-converting enzyme 2 (ACE2), as SARS-CoV-1 for viral entry [3,16-18]. Due to its role in virus entry, the $S$ protein has been the target for the generation of monoclonal antibodies (mAb).

In our previous work, we used five different fragments of SARS-CoV-1 S protein to immunise rabbits.
A fragment corresponding to residues 1029 to 1192 in the $\mathrm{S} 2$ subunit of SARS-CoV-1 was found to stimulate neutralising antibodies against SARS-CoV-1 [19]. This fragment was subsequently used to generate a panel of murine mAbs with their respective binding domains characterised and described in Lip et al. [20]. One of them, mAb $1 A 9$, which binds to the $S$ protein through a recently identified epitope within the $S_{2}$ subunit at amino acids 1111-1130, has the ability to bind and cross-neutralise pseudotyped viruses expressing the $\mathrm{S}$ protein of human SARS-CoV-1, civet SARS-CoV and bat SL-CoV strains [21]. In this study, we aim to verify if the sequence of the immunogen used to generate $\mathrm{mAb} 1 \mathrm{~A} 9$, as well as three other $\mathrm{mAbs}$, is conserved in different coronaviruses and if these mAbs bind to the $\mathrm{S}$ protein of SARS-CoV-2 expressed in mammalian cell lines. Importantly, mAb $1 A 9$ is investigated for its ability to detect the $\mathrm{S}$ protein in SARS-CoV-2 infected cells and purified $S$ protein in a sandwich ELISA format when paired with another mAb binding to the $\mathrm{S}_{1}$ subunit of SARS-COV-2.

\section{Methods}

\section{Cells}

Vero E6 and COS-7 cells were purchased from the American Type Culture Collection (Manassas, VA, United States) and cultured in Dulbecco's Modified Eagle's Medium (DMEM; Thermo Fisher Scientific, Waltham MA, United States) supplemented with 10\% fetal bovine serum (FBS; HyClone, Logan, UT, United States), 100 units $/ \mathrm{mL}$ penicillin and $100 \mu \mathrm{g} / \mathrm{mL}$ streptomycin (Thermo Fisher Scientific). 293FT cells were purchased from Invitrogen (Carlsbad, CA, United States) 
Pairwise amino-acid identity across relevant coronaviruses in the sequence fragment of the spike glycoprotein S2 subunit recognised by monoclonal antibody $1 \mathrm{~A} 9$ or the sequence of the full spike glycoprotein

\begin{tabular}{|c|c|c|c|c|c|c|c|c|}
\hline \multirow{2}{*}{ Query/reference } & \multicolumn{8}{|c|}{ Pairwise amino-acid identity (\%) } \\
\hline & SARS-CoV-2 & BatRaTG13 & SARS-CoV-1 & MERS & $\mathrm{OC}_{43}$ & $\mathrm{HKU}_{1}$ & $229 \mathrm{E}$ & NL63 \\
\hline \multicolumn{9}{|c|}{ Fragment region of spike S2 } \\
\hline SARS-Co-V2 & 100.00 & SB & SB & SB & SB & SB & SB & SB \\
\hline BatRaTG13 & 99.40 & 100.00 & SB & SB & SB & SB & SB & SB \\
\hline SARS & 93.10 & 92.50 & 100.00 & SB & SB & SB & SB & SB \\
\hline MERS & 39.00 & 39.00 & 39.00 & 100.00 & SB & SB & SB & SB \\
\hline $0 C_{43}$ & 39.00 & 39.00 & 38.40 & 51.20 & 100.00 & SB & SB & SB \\
\hline $\mathrm{HKU}_{1}$ & 32.70 & 32.70 & 30.80 & 50.60 & 68.40 & 100.00 & SB & SB \\
\hline $229 \mathrm{E}$ & 30.80 & 30.20 & 32.10 & 31.50 & 29.70 & 30.40 & 100.00 & SB \\
\hline NL63 & 30.80 & 30.20 & 30.20 & 32.10 & 31.60 & 33.50 & 64.20 & 100.00 \\
\hline \multicolumn{9}{|l|}{ Full spike protein } \\
\hline SARS-CoV-2 & 100.00 & SB & SB & SB & SB & SB & SB & SB \\
\hline BatRaTG13 & 97.70 & 100.00 & SB & SB & SB & SB & SB & SB \\
\hline SARS-CoV-1 & 77.80 & 78.20 & 100.00 & SB & SB & SB & SB & SB \\
\hline MERS & 35.40 & 35.40 & 35.20 & 100.00 & SB & SB & SB & SB \\
\hline OC43 & 37.30 & 37.10 & 36.90 & 39.50 & 100.00 & SB & SB & SB \\
\hline $\mathrm{HKU}_{1}$ & 35.20 & 35.30 & 35.00 & 39.00 & 67.00 & 100.00 & SB & SB \\
\hline $229 \mathrm{E}$ & 41.70 & 41.50 & 41.80 & 41.80 & 43.50 & 43.50 & 100.00 & SB \\
\hline NL63 & 36.30 & 36.20 & 36.20 & 35.40 & 39.70 & 37.80 & 64.70 & 100.00 \\
\hline
\end{tabular}

MERS: Middle East respiratory syndrome; SARS-CoV-1: severe acute respiratory coronavirus; SARS-CoV-2: severe acute respiratory coronavirus; SB: shown below. High to low pairwise amino-acid identity are coloured coded respectively by contrasting green to red backgrounds.

The sequence identity is not affected by the order in which paired sequences are compared so only one-way comparisons are shown to avoid redundancies; the abbreviation 'SB' is used when the pairwise amino-acid identity in question is already shown in a further cell of the table.

and grown in DMEM supplemented with $10 \%$ FBS, 100 units $/ \mathrm{mL}$ penicillin, $100 \mu \mathrm{g} / \mathrm{mL}$ streptomycin and $500 \mu \mathrm{g} / \mathrm{mL}$ geneticin (Thermo Fisher Scientific). Cells were maintained at $37^{\circ} \mathrm{C}$ with $5 \% \mathrm{CO}_{2}$.

\section{Purification of monoclonal antibody 1 A9}

The hybridoma for $\mathrm{mAb} 1 \mathrm{~A} 9$ was previously generated [20]. All mAbs were purified from cell culture supernatants using HiTrap protein G HP affinity columns (GE Healthcare, Chicago, IL, United States) and stored at $-80^{\circ} \mathrm{C}$. The purity of the mAb was confirmed by sodium dodecyl sulphate-polyacrylamide gel electrophoretic (SDS-PAGE) analysis. The concentration of the purified $\mathrm{mAb}$ was determined using the Coomassie Plus protein assay reagent (Thermo Fisher Scientific).

\section{Plasmids for expression of full spike protein and fragments}

SARS-CoV-2 S-protein-expressing plasmids were codon-optimised and generated by gene synthesis (Bio Basic Asia Pacific, Singapore) according to GenBank accession number: QHD43416.1. One plasmid is for expressing untagged full-length $\mathrm{S}$ protein while the other is for expressing a Myc-tagged S-protein fragment consisting of residues 1048-1206 (SARS-CoV-2 numbering). The $\mathrm{pXJ} 40-\mathrm{Myc}$ expression vector was used as an empty vector control and $\mathrm{pXJ} 40-\mathrm{Myc}-\mathrm{HBcAg}$ plasmid expressing Myc-tagged hepatitis $B$ virus core antigen ( $\mathrm{HBCAg}$ ) was used as a negative control.

\section{Transient transfection and western blot analysis}

293 FT cells were seeded onto $6-\mathrm{cm}$ dishes 24 hours before transient transfection using X-tremeGENE HP DNA transfection reagent (Roche, Basel, Switzerland) according to the manufacturer's protocol. At 24 hours post-transfection, cells were harvested, spun down by centrifugation and washed with cold phosphate buffered saline (PBS) twice. Cells were then resuspended in $2 \times$ Laemmli sample buffer, boiled and sonicated. Clarified supernatant containing the protein of interest was obtained by spinning down the cell lysate at $13,000 \mathrm{rpm}$ at $4^{\circ} \mathrm{C}$ to remove the cell debris and further analysed by western blot (WB) analysis. Equal amounts of total cell lysates were loaded per lane and resolved using electrophoresis on SDS-PAGE gels and transferred onto nitrocellulose membrane (BioRad, Hercules, CA, United States). The membrane was blocked in $5 \%$ skimmed milk in Tris-buffered saline with $0.05 \%$ Tween 20 (TBST) for 1 hour at room temperature (RT) and incubated with primary antibodies at $4^{\circ} \mathrm{C}$ overnight. After the membrane was washed with TBST, it was incubated with a horseradish peroxidase (HRP)-conjugated secondary antibody (Thermo Fisher Scientific) at RT for 1 hour. The membrane was then washed with TBST again and bound antibodies visualised with enhanced chemiluminescence substrate (Thermo Fisher Scientific) using ChemiDoc MP Imaging System (Bio-Rad). 


\section{FIGURE 2}

Monoclonal antibodies expected to target a SARS-CoV-2 S protein S2 fragment, (A) hybridise to the peptide fragment in western blot and (B) recognise cells expressing the peptide as shown by immunofluorescence

A.

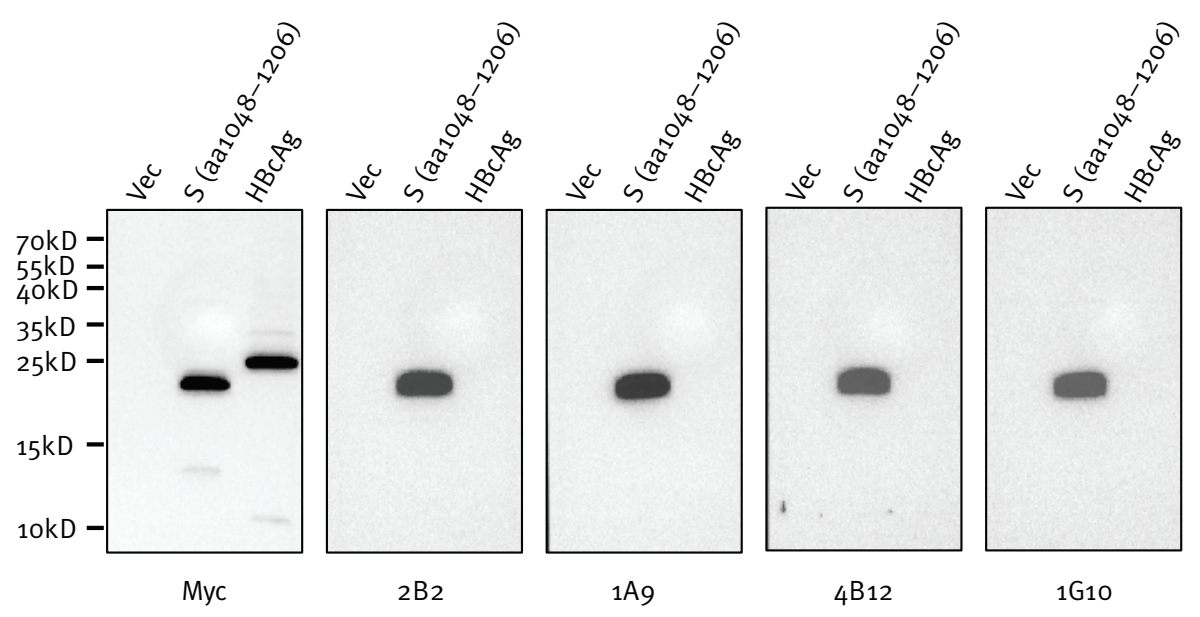

B.

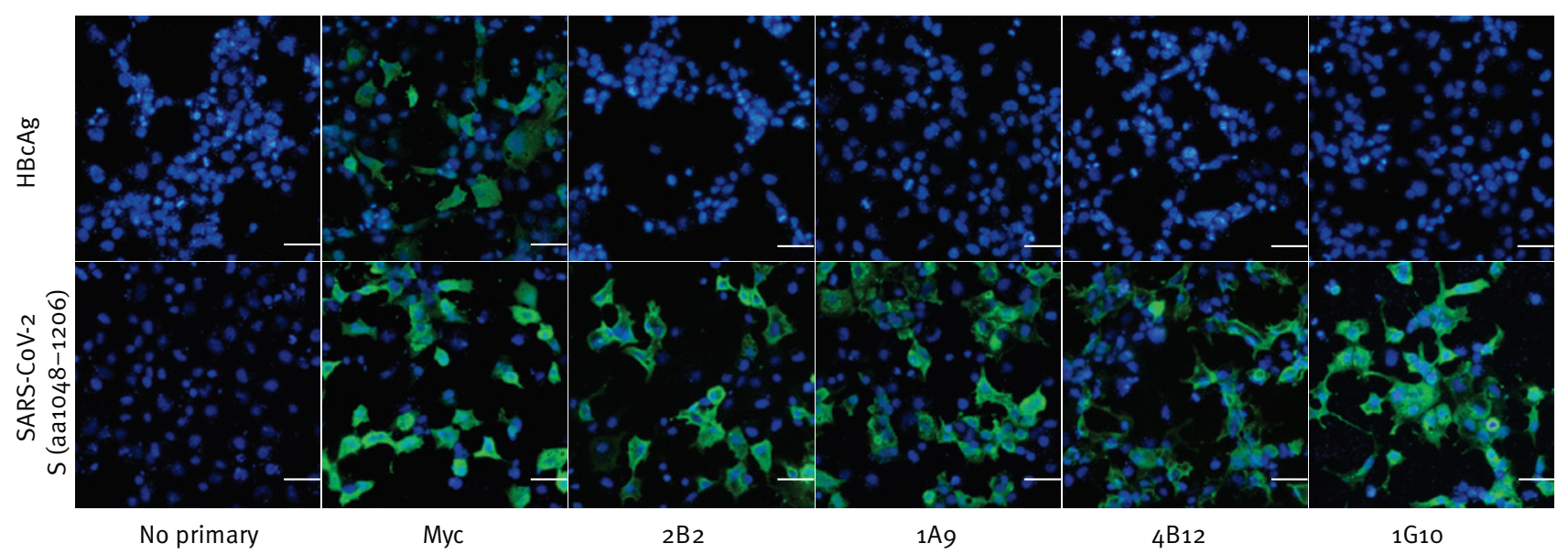

aa: amino acid; HBcAg: hepatitis B virus core antigen; DAPI: 40,6-diamidino-2-phenylindole; SARS-CoV-2: severe acute respiratory syndrome coronavirus 2; S protein: spike protein; vec: $\mathrm{pXJ40-Myc} \mathrm{expression} \mathrm{vector} \mathrm{used} \mathrm{as} \mathrm{an} \mathrm{empty} \mathrm{vector} \mathrm{control.}$

A. Each photo depicts a western blot using the primary antibody indicated below it (Myc, 2B2, 1A9, 4B12, or 1G10). Empty vector-transfected 293 FT cell products are on the lanes labelled as 'Vec', while products of 293FT cells expressing Myc-tagged SARS-CoV-2 S protein fragment (aa 1048-1206; SARS-CoV-2 numbering) or Myc-tagged HBcAg are on the lanes respectively labelled ' $S$ ' or 'HBcAg'. Primary antibodies were labelled with horseradish peroxidase-conjugated secondary antibodies. A ladder indicative of the molecular weights in kD of the proteins relative to their vertical position on the blots, is indicated on the left of the panel.

B. Each photo depicts an immunofluorescence assay using either no primary antibody, or the primary antibody indicated below it (Myc, 2B2, $1 \mathrm{~A} 9,4 \mathrm{~B} 12$, or $1 \mathrm{G} 10$ ). Immunofluorescence assay results of COS7 cells expressing Myc-tagged HBcAg (top photos) or a Myc-tagged fragment (aa1048-1206) of SARS-CoV-2 S protein (bottom photos) using the indicated primary antibodies, followed by Alexa Fluor 488-conjugated secondary antibody (green) are shown. Cell nuclei were counterstained with DAPI (blue). Scale bar $=50 \mu \mathrm{m}$.

\section{Transient transfection and}

\section{immunofluorescence analysis}

For immunofluorescence (IF) analysis, COS-7 cells on glass coverslips were transfected as above and fixed at 24 hours post-transfection in $4 \%$ paraformaldehyde for $10 \mathrm{~min}$ at RT followed by permeabilisation with $0.2 \%$ Triton X-10o (Sigma-Aldrich, St. Louis, MO, United States) for $5 \mathrm{~min}$. Fixed cells were then blocked with PBS containing $10 \%$ FBS for $30 \mathrm{~min}$ at RT. Cells were immunolabelled for 1 hour at RT with the indicated murine $\mathrm{mAb}$ and $45 \mathrm{~min}$ with Alexa Fluor 488-conjugated goat anti-mouse IgG antibody (Life Technologies, Carlsbad, CA, United States). Immunolabelled coverslips were counterstained with 40,6-diamidino-2-phenylindole (DAPI; Sigma-Aldrich), and mounted using ProLong Gold Antifade Mountant (Molecular Probes, Eugene, OR, United States). Images were acquired with Olympus CKX53 microscope using Olympus (Tokyo, Japan) LCAch $\mathrm{N} 20 \times / 0.40$ iPC objective lens and Olympus DP27 colour camera with Olympus cellSens software. Each channel was collected separately, with images at $1024 \times 1024$ pixels.

\section{ELISA}

Whole ectodomain of SARS-CoV-2 S protein with His Tag (Sino Biological Inc., Beijing, China; catalogue 


\section{FIGURE 3}

Antibodies expected to target SARS-CoV-2 S protein, (A) hybridise to the denatured protein in western blot, (B) bind to the protein in ELISA and $(\mathrm{C})$ recognise cells expressing the protein as shown by immunofluorescence

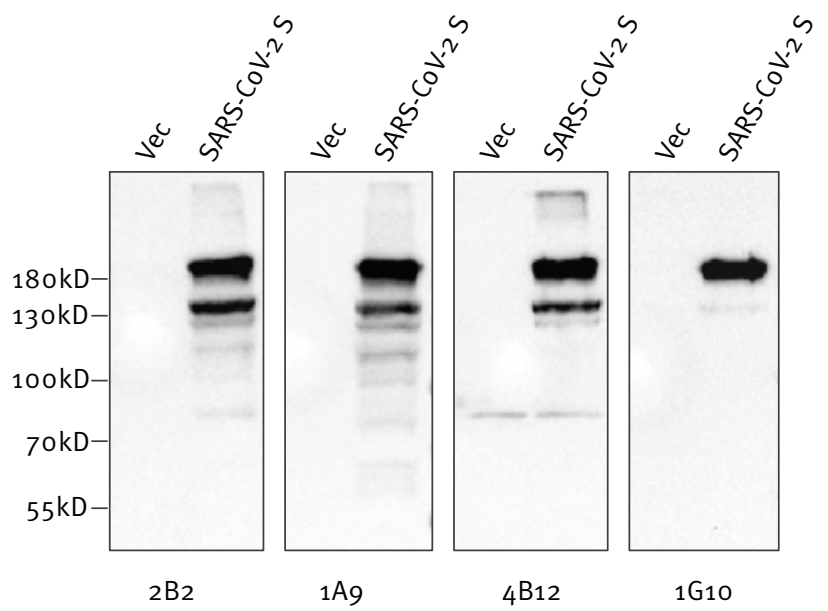

C.

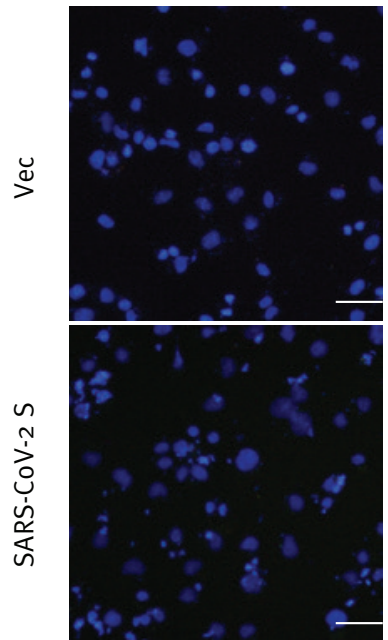

No primary
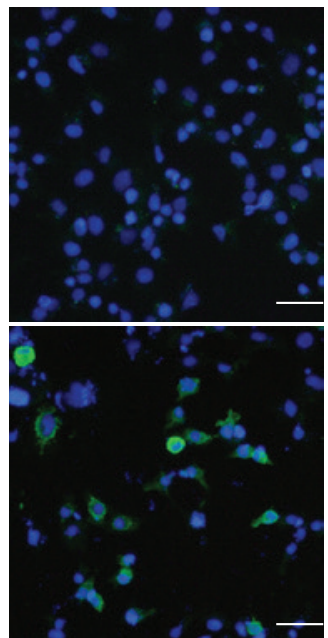

$2 \mathrm{~B} 2$
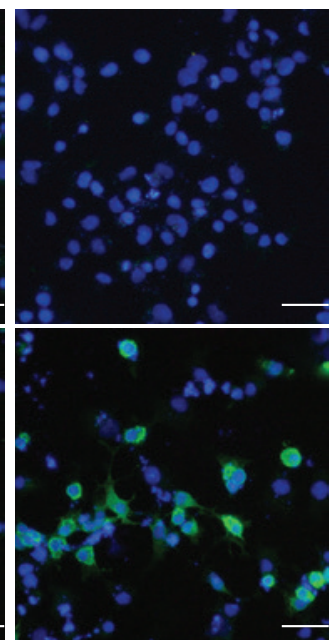

$1 \mathrm{Ag}$

B.

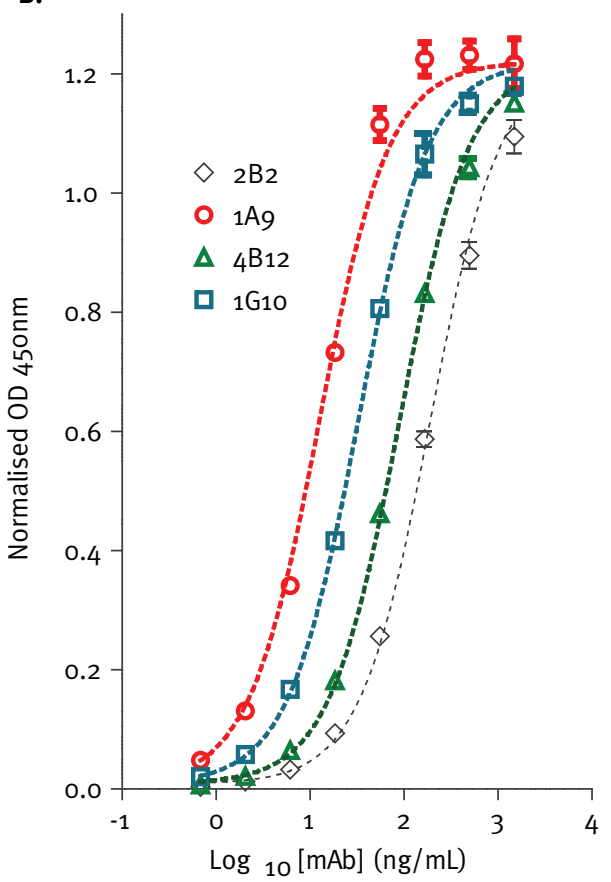

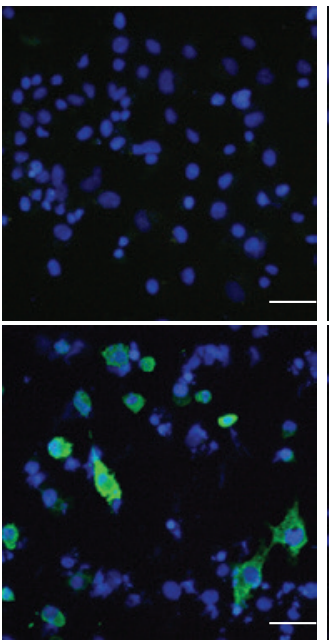

$4 \mathrm{~B} 12$

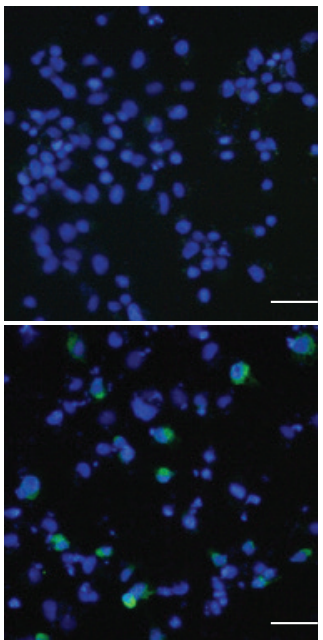

$1 \mathrm{G} 10$

DAPI: 4D,6-diamidino-2-phenylindole; mAb: monoclonal antibody: OD: optical density; SARS-CoV-2: severe acute respiratory syndrome coronavirus 2; S protein: spike protein; vec: $\mathrm{pXJ} 40-$ Myc expression vector used as an empty vector control.

A. Each photo depicts a western blot using the primary antibody indicated below it (2B2, $1 \mathrm{~A} 9,4 \mathrm{~B} 12$, or $1 \mathrm{G} 10)$. Empty vector-transfected 293FT cell products are on the lanes labelled as 'Vec', while products of 293FT cells expressing full-length SARS-CoV-2 S protein are on the lanes labelled 'SARS-CoV-2 S'. Primary antibodies were labelled with horseradish peroxidase-conjugated secondary antibodies. A ladder indicative of the molecular weights in $\mathrm{kD}$ of the proteins relative to their vertical position on the blots, is indicated on the left of the panel.

B. The abilities of $2 \mathrm{~B}_{2}, 1 \mathrm{~A} 9,4 \mathrm{~B} 12$ and $1 \mathrm{G} 10$ monoclonal antibodies to bind to SARS-CoV-2 S protein was determined by ELISA. Individual wells were coated with $20 \mathrm{ng}$ of SARS-CoV-2 S protein and incubated with serially diluted mAbs as indicated. A representative plot from three independent experiment is show for each antibody and error bars correspond to standard deviations of each mAb experiment carried out in triplicates.

C. Each photo depicts an immunofluorescence assay using either no primary antibody, or the primary antibody indicated below it (Myc, 2B2, $1 \mathrm{~A} 9,4 \mathrm{~B} 12$, or $1 \mathrm{G} 10$ ). Immunofluorescence analysis was performed on empty vector-transfected COS-7 cells (top photos) and cells expressing full-length SARS-CoV-2 S protein (bottom photos). The indicated primary antibodies were used followed by Alexa Fluor 488-conjugated secondary antibody (green). Cell nuclei were counterstained with DAPI (blue). Scale bar $=50 \mu \mathrm{m}$. 


\section{FIGURE 4}

Performance of monoclonal antibody 1A9 for detection of (A) $\mathrm{S}$ protein in a sandwich ELISA format and (B) SARSCoV-2 infected cells

A.

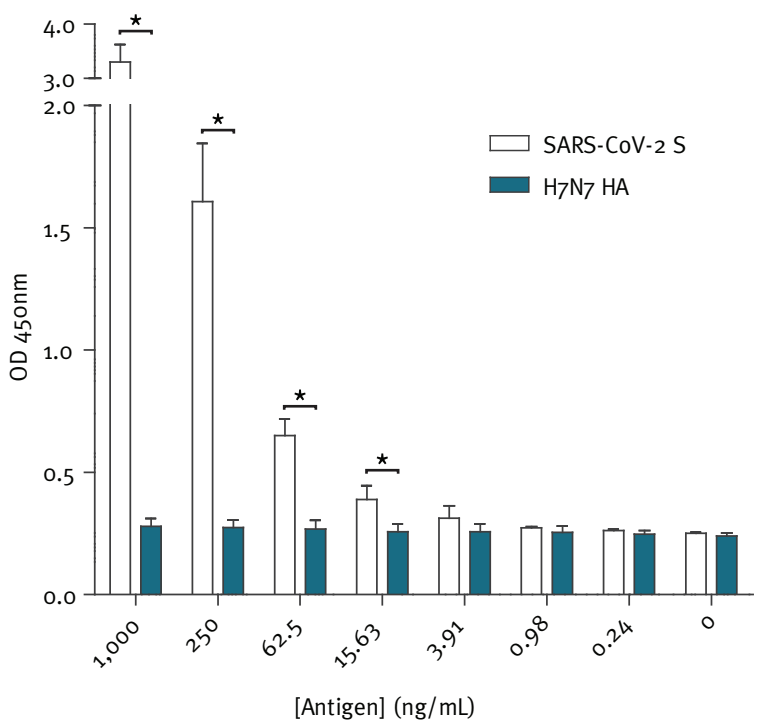

B.

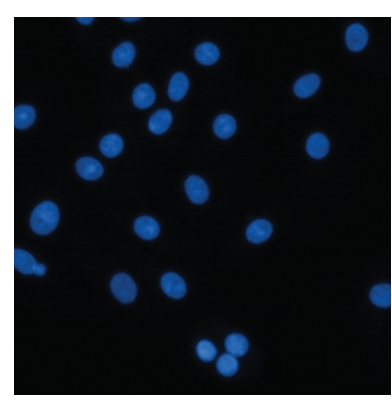

Mock

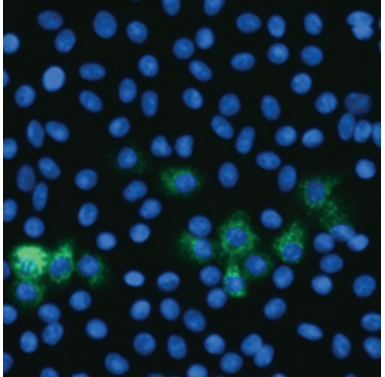

SARS-COV-2
$\mathrm{HA}$ : haemagglutinin; $\mathrm{H}_{7} \mathrm{~N}_{7}$ : influenza $\mathrm{A}\left(\mathrm{H}_{7} \mathrm{~N} 7\right)$; mAb: monoclonal antibody; MOI: multiplicity of infection; OD: optical density; SARSCoV-2: severe acute respiratory syndrome coronavirus 2.

A. Sandwich ELISA assay to determine $m A b 1 A 9$ ability to pair with the human mAb CR 3022 for the detection of a His-tagged SARS-CoV-2 spike protein. $1 \mathrm{~A} 9$ and CR3022 were used as capture and detector antibodies respectively. His-tagged HA protein of influenza $\mathrm{A}\left(\mathrm{H}_{7} \mathrm{~N}_{7}\right)$ virus was used as a negative control. Averaged readings across three replicate experiments are presented. Error bars represent standard deviations across the three replicate experiments. Asterisks indicate significantly increased binding of the antibody pairs to SARS-CoV-2 S protein compared to influenza $\mathrm{A}\left(\mathrm{H}_{7} \mathrm{~N} 7\right) \mathrm{HA}$ at $\mathrm{p}<0.05$.

B. Vero E6 cells were mock-infected (left panel) or infected with SARS-CoV-2 (right panel; MOI of 1). At 24 hour post infection, the cells were stained with $\mathrm{mAb}_{1 \mathrm{~A}}(5 \mu \mathrm{g} / \mathrm{mL})$ followed by Alexa Fluor 488-conjugated secondary antibody (green). Nuclei were counterstained with 40,6-diamidino-2-phenylindole (DAPI; blue). number: 40589-Bo8V1) was diluted with coating buffer $\left(0.1 \mathrm{M} \mathrm{NaHCO}_{3}, 34 \mathrm{mM} \mathrm{Na}_{2} \mathrm{CO}_{3}\right.$ ) and a total of $20 \mathrm{ng}$ of protein was loaded into individual wells of a 96 well plate (Nunc, Roskilde, Denmark) and allowed to coat overnight at $4^{\circ} \mathrm{C}$. Plates were then washed four times with $0.05 \%$ Tween 20 in PBS (PBST) and blocked with $5 \%$ bovine serum albumin (BSA)/PBST for $30 \mathrm{~min}$ before murine antibodies serially diluted with blocking buffer were added to desired wells for 1 hour. Plate were washed four times with PBST before incubation for 1 hour with HRP-conjugated goat anti-mouse IgG (Thermo Fisher Scientific) secondary antibodies diluted in blocking buffer, and washed four times with PBST. Visualisation of bound secondary antibodies was done by the addition of 3,3',5,5'-tetramethylbenzidine (TMB) substrate (Thermo Fisher Scientific) for $5 \mathrm{~min}$ in the absence of light and the reaction was stopped with $2 \mathrm{M}$ sulphuric acid. Optical density at $450 \mathrm{~nm}$ (OD450nm) was determined by a Tecan (Männedorf, Switzerland) Infinite M1000 reader and normalised OD450nm was obtained by subtracting background absorbances determined in BSA coated wells.

\section{Production of monoclonal antibody CR3022}

The human mAb CR3022 was expressed in a similar manner as previously described [22]. The variable heavy (VH; GenBank accession number: DQ168569) and variable light (VL; GenBank accession number: $D Q 168570)$ genes of CR3022 were generated by gene synthesis (Bio Basic Asia Pacific) and cloned into pFUSEss-CHIg-hlgG1 and pFUSE2ss-CLIg-hK cloning vectors (InvivoGen, San Diego, CA, United States) respectively. Transfection of suspension FreeStyle 293 cells (Thermo Fisher Scientific) and purification of antibodies by fast protein liquid chromatography is as described in our previous study [23].

\section{Sandwich ELISA}

Mab 1 A9 was diluted with coating buffer (0.1 $\mathrm{M} \mathrm{NaHCO}_{3}$, $34 \mathrm{mM} \mathrm{Na}_{2} \mathrm{CO}_{3}$ ) and $0.1 \mu \mathrm{g}$ of antibody was coated onto individual wells of a Maxisorp flat-bottom plate (Nunc) overnight at $4^{\circ} \mathrm{C}$. The plate was washed three times with PBST before blocking was done using 5\% BSA/ PBST at $37^{\circ} \mathrm{C}$ for $60 \mathrm{~min}$. Dilutions of His-tagged full length SARS-CoV-2 S protein (Sino Biological Inc., catalogue number: 40589-Bo8V1) and His-tagged $\mathrm{H}_{7} \mathrm{~N}_{7}-\mathrm{HA}$ (Sino Biological Inc., catalogue number: 11082-Vo8B) were added to desired wells and incubated at $37^{\circ} \mathrm{C}$ for 90 min followed by three washes with PBST. $100 \mu \mathrm{L}$ of CR3022 antibody was added at a concentration of $1 \mu \mathrm{g} / \mathrm{mL}$ and incubated at $37^{\circ} \mathrm{C}$ for 60 min followed by three PBST washes before HRP-conjugated goat antihuman IgG (Thermo Fisher Scientific) was added for $60 \mathrm{~min}$ at $37^{\circ} \mathrm{C}$. Finally, after three PBST washes, TMB (Sigma-Aldrich) was added for $5 \mathrm{~min}$ and the reaction was stopped by $2 \mathrm{M}$ sulphuric acid. The OD450nm was determined by a Tecan Infinite M10oo reader. Statistical analyses were performed using an unpaired, one-tailed Student's t-test with Welch's correction for unequal variances. $p$ values $<0.05$ were considered statistically significant. 
Virus infection and immunofluorescence

All works with live virus were performed in the biosafety level (BSL)3 facility at the Public Health Agency of Sweden. Vero-E6 cells were infected with SARS-CoV-2 (SARS-CoV-2-Iso/01/human/2020/SWE; GenBank accession number: MTo93571) at a multiplicity of infection (MOI) of one in DMEM $2 \%$ FBS (Thermo Fisher Scientific). At 24 hour post-infection, cells were fixed with chilled methanol/acetone and the cells were kept at $-20^{\circ} \mathrm{C}$ overnight. Cells were then stained using $\mathrm{mAb} 1 \mathrm{Ag}$ at $5 \mu \mathrm{g} / \mathrm{mL}$ at $37^{\circ} \mathrm{C}$ for $30 \mathrm{~min}$ in IF buffer (BSA $0.2 \%$, Triton $\times 1000.1 \%$ in $\mathrm{PBS}, \mathrm{pH} 7.4$ ). The cells were washed three times with PBS and incubated, subsequently with Alexa Fluor 488-conjugated goat antimouse IgG antibody (Thermo Fisher Scientific) in IF buffer containing DAPI for an additional $30 \mathrm{~min}$. Cells were washed three times with PBS before visualisation and image acquisition with fluorescent microscopy.

\section{Bioinformatics analysis}

$\mathrm{S}$ protein reference sequences for SARS-CoV-1, SARSCoV-2, batRaTG13, Middle East respiratory syndrome (MERS) and human common-cold coronaviruses $229 \mathrm{E}$, NL63, OC43 and HKU1 were downloaded from the National Center for Biotechnology Information (NCBI). A multiple sequence alignment was created with multiple alignment using fast Fourier transform (MAFFT) using the slow but accurate L-INS-I parameter settings [24] and the alignment curated, cut to the target region 1029-1192 (SARS-CoV-1 numbering) and visualised with Jalview [25]. We used Molecular Evolutionary Genetics Analysis (MEGA) X [26] to calculate the number of amino-acid differences for all sequence pairs in the alignment of the mAb target region and the full $S$ protein normalised by the length of the aligned sequence of the respective reference protein to obtain per cent amino acid identities.

To determine SARS-CoV-2 sequence diversity in the $S$ protein within the current pandemic, 230 human and environmental viral sequences were downloaded from GISAID's EpiCoV database on 1 March 2020. We gratefully acknowledge the authors, originating and submitting laboratories of the sequences on which this part of the research is based. The list is detailed in Supplementary Table 1. The nt sequences were searched with basic local alignment search tool (BLAST)X against the reference S protein. 174 hits covered the full length of the $S$ protein and amino-acid mutations were counted and tabulated using a custom Perl script (Supplementary Table 2).

\section{Ethical statement}

Ethical approval was not required for this study.

\section{Results}

An immunogenic domain in the S2 subunit of SARS-CoV-1 is highly conserved in

\section{SARS-CoV-2 but not in MERS and common cold $\mathrm{HCoV}$}

Sequence alignment of the S2 fragment corresponding to residues 1029 to 1192 shows that this fragment, which encompasses the heptad repeat (HR)2 but not HR1, is highly conserved in SARS-CoV-1 and SARS-CoV-2 (Figure 1). When compared with additional reference sequences from bat RaTG13 (closest bat precursor), MERS and human common cold coronaviruses $229 \mathrm{E}$, NL63, OC43 and HKU1 (Figure 1), it becomes apparent that the amino-acid identity between SARS-CoV-2 and SARS-CoV-1 is much higher in this region (93\%, Table) than over the full protein length (78\%, Table) and the similarity drops sharply ( $40 \%$ in this region) when considering MERS and the other coronaviruses infecting humans regularly.

We also studied the sequence diversity across 174 SARS-CoV-2 $S$ proteins derived from nt sequences shared via the GISAID platform [27]. Only four aminoacid mutations were found within the putative antibody-binding region compared with 30 mutations over the full length protein (Supplementary Table 2). Two of these four amino-acid mutations are from a sequence flagged in GISAID's EpiCoV database as lower quality due to many undetermined bases.

Four murine monoclonal antibodies bind to a fragment of the spike protein of SARS-CoV-2 Four mAbs with distinct binding profiles to SARS-CoV-1, as previously mapped by internal deletion mutagenesis study, were selected for testing to determine if they cross-react with SARS-CoV-2. A fragment containing residues 1048 to 1206 of SARS-CoV-2 S protein was expressed in $293 \mathrm{FT}$ cells via transient transfection and WB analysis was performed using the four mAbs, namely $2 B 2,1 A 9,4 B 12$ and $1 G 10$. As shown in Figure $2 A$, all four mAbs detected this fragment of SARSCoV-2, which is consistent with the sequence alignment shown in Figure 1. Due to the easy detachment of 293FT cells, COS-7 cells were used for IF assay instead. IF analysis performed on transiently transfected COS-7 cells showed binding of the four $\mathrm{mAbs}$ to this $\mathrm{S}$ protein fragment of SARS-CoV-2 (Figure 2B). These interactions are also specific for the SARS-COV-2 S protein (10481206) fragment as all four mAbs did not show binding to the negative control $\mathrm{HBCAg}$.

Four murine monoclonal antibodies bind to the full-length S protein of SARS-CoV-2

Next, the full-length S protein of SARS-CoV-2 was overexpressed in 293FT and COS-7 cells and detected with each of the mAbs using WB and IF analyses. As shown in Figure 3, all four mAbs bound to the full-length $S$ protein of SARS-CoV-2 (Figure 3A).

The binding of these mAbs to recombinant purified $S$ protein was also determined using indirect ELISA where different concentrations of antibodies were used for binding. Binding to $\mathrm{S}$ protein was observed for all four mAbs with $1 \mathrm{Ag}$ showing the strongest binding (Figure 
3B). Similarly, all four mAbs bound to the full-length $\mathrm{S}$ protein of SARS-CoV-2 when tested via IF (Figure 3C). Collectively our data demonstrates the ability of all four mAbs to bind full-length $\mathrm{S}$ protein in both its native and denatured forms.

Utility of monoclonal antibody $1 \mathrm{Ag}$ for detection of $\mathrm{S}$ protein in a sandwich ELISA format and in SARS-CoV-2 infected cells

Based on indirect ELISA data, $\mathrm{mAb} 1 \mathrm{~A} 9$ has the strongest binding to $S$ protein when compared with the other three mAbs. Hence, a sandwich ELISA was performed to determine if it can be paired with the human $\mathrm{mAb}$ CR3022 which is known to bind to the $\mathrm{S}_{1}$ subunit of SARS-CoV-2. As shown in Figure $4 \mathrm{~A}$, recombinant $\mathrm{S}$ protein was detected at $15.6 \mathrm{ng} / \mathrm{mL}$ and above when $1 \mathrm{~A} 9$ was used as a capture antibody and $\mathrm{CR}_{3022}$ was used as a detector antibody. Since the $\mathrm{S}$ protein was His-tagged, a His-tagged haemagglutinin (HA) protein of influenza A virus was used to check for specificity of binding. The absorbance readings in the presence of $S$ protein were significantly higher than that in the presence of HA for protein concentrations of $15.6 \mathrm{ng} / \mathrm{mL}$ and above.

Next, 1 A9 was tested on SARS-CoV-2-infected Vero-E6 cells. As shown in Figure $4 B, m A b 1 A 9$ stained a considerable number of SARS-CoV-2-infected cells at 24 hours post-infection showing that it is sensitive enough to detect the expression of $\mathrm{S}$ protein during infection.

\section{Discussion}

Numerous mAbs against the $\mathrm{S}$ protein of SARS-CoV-1 have been generated for research and diagnostic assay development. Some of these may be able to cross-react with the S protein of SARS-CoV-2 and serve as tools to aid research on this newly emerged virus. In this current study, an immunogenic domain in the S2 subunit of SARS-CoV-1 was found to be highly conserved in multiple strains of SARS-CoV-2 (Figure 1 and Table). Consistently, WB and IF analyses showed that four different mAbs generated using this SARS-CoV-1 domain were cross-reactive against the $S$ protein of SARSCoV-2 (Figures 2 and 3).

Recent cross-reactivity studies have evaluated SARSCoV-1 neutralising antibodies that bind to the RBDcontaining S1 subunit. Although both SARS-CoV-1 and SARS-CoV-2 use ACE2 as a receptor for viral entry $[3,16]$, several SARS-CoV-1 RBD-directed mAbs did not cross-react with SARS-CoV-2 RBD $[28,29]$. Interestingly, CR3022, which was isolated from a SARS convalescent patient [22], showed cross-reactivity to SARS-CoV-2 RBD and recognises an epitope that does not overlap with the ACE2 binding site [28]. Among the four mAbs tested in this study, indirect ELISA showed that $1 \mathrm{~A} 9$ binds strongest to the S protein of SARS-CoV-2 (Figure $3 B)$. To determine if $1 A 9$ is useful for detection of $S$ protein in a sandwich ELISA, it was paired with $\mathrm{CR}_{3022}$ since $1 \mathrm{~A} 9$ binds to $\mathrm{S} 2$ subunit while $\mathrm{CR}_{3022}$ binds to
S1 subunit. As would be expected, these two antibodies can be paired to detect $S$ protein from $15.6 \mathrm{ng} / \mathrm{mL}$ (Figure $4 \mathrm{~A}$ ). In addition, $\mathrm{mAb} 1 \mathrm{~A} 9$ stained a considerable number of SARS-CoV-2-infected cells at 24 hours post-infection showing that it is sensitive enough to detect the expression of $S$ protein during infection (Figure $4 \mathrm{~B}$ ). Thus, mAbs $1 \mathrm{Ag}$ will be useful for studying the kinetics of SARS-CoV-2 replication in vitro and development of diagnostic assays for COVID-19. It is noteworthy that cytotoxic T-lymphocyte (CTL) epitopes also reside at residues $884-891$ and $1116-1123$ within the S2 subunit of SARS-CoV-1 [30]. Interestingly, the latter CTL epitope overlaps with the epitope recognised by $\mathrm{mAb} 1 \mathrm{Ag}$ [21]. Hence, the $\mathrm{S} 2$ subunit may serve as an important antigen for inducing both humoral as well as cell-mediated immunity against SARS-CoV-1 and SARS-CoV-2.

To our knowledge, this is the first study showing that mAbs targeting the S2 domain of SARS-CoV-1 can cross-react with SARS-CoV-2 and this observation is consistent with the high sequence conservation in the S2 subunit. The ability of these antibodies, particularly $1 \mathrm{~A} 9$, to detect SARS-CoV-2 S protein in indirect and sandwich ELISAs demonstrate their utility for detection of SARS-CoV-2 infections in a public health setting. Whether or not the current sensitivity of these antibodies are sufficient for robust detection of SARS-CoV-2 infections in a clinical setting and how they compare to existing PCR-based detection remains to be determined. Successful development of these antibodies into a point of care diagnostic kit will provide a complementary approach to existing detection methods. Besides the mAbs characterised here, several other mAbs have been reported to bind to epitopes in the S2 subunit of SARS-CoV-1 [31-33]. Thus, it will be important to determine if these mAbs can also cross-react with SARS-CoV-2.

\section{Acknowledgements}

The work performed in NUS/NUHS was supported by NUHS Research Office under Project Number NUHSRO/2020/033/ R05+5/CORONAVIRUS/LOA (WBS R-571-000-071-733). The work performed in IMCB and BII was also supported by $A^{\star}$ STAR through intramural funding and an $A^{*} C R U S E$ gap funding (ACCL/19-GAP064-R20H-F).

\section{Conflict of interest}

WJH and YJT declare that they are involved in the licensing of mAbs $1 \mathrm{~A} 9,1 \mathrm{G}_{10}, 4 \mathrm{~B}_{12}$ and $2 \mathrm{~B}_{2}$ to commercial companies as research or diagnostic reagents. The other authors have declared that no competing interests exist.

\section{Authors' contributions}

Zheng Z - Designed experiments, performed experiments, analysed and organised data, wrote the manuscript.

Monteil, VM - Designed experiments, performed experiments, analysed and organised data, wrote the manuscript. 
Maurer-Stroh, S - Designed experiments, performed experiments, analysed and organised data, wrote the manuscript.

Yew CW - Designed experiments, performed experiments.

Leong C - Designed experiments, performed experiments.

Mohd-Ismail NK - Designed experiments, performed experiments, analysed and organised data, wrote the manuscript.

Cheyyatraivendran Arularasu S - Designed experiments, performed experiments.

Chow VTK - Analysed and organised data.

Lin RTP - Analysed and organised data.

Mirazimi A- Designed experiments, performed experiments, analysed and organised data, wrote the manuscript.

Hong WJ- Analysed and organised data.

Tan YJ- Designed experiments, performed experiments, analysed and organised data, wrote the manuscript.

\section{References}

1. Drosten C, Günther S, Preiser W, van der Werf S, Brodt $\mathrm{HR}$, Becker S, et al. Identification of a novel coronavirus in patients with severe acute respiratory syndrome. N Engl J Med. 2003;348(20):1967-76. https://doi.org/10.1056/NEJMoa030747 PMID: 12690091

2. Ksiazek TG, Erdman D, Goldsmith CS, Zaki SR, Peret T, Emery $\mathrm{S}$, et al. , SARS Working Group. A novel coronavirus associated with severe acute respiratory syndrome. N Engl J Med. 2003;348(20):1953-66. https://doi.org/10.1056/NEJMoa030781 PMID: 12690092

3. Zhou P, Yang XL, Wang XG, Hu B, Zhang L, Zhang W, et al. A pneumonia outbreak associated with a new coronavirus of probable bat origin. Nature. 2020;579(7798):270-3. https:// doi.org/10.1038/S41586-020-2012-7 PMID: 32015507

4. Jiang S, Du L, Shi Z. An emerging coronavirus causing pneumonia outbreak in Wuhan, China: calling for developing therapeutic and prophylactic strategies. Emerg Microbes Infect. 2020;9(1):275-7. https://doi.org/10.1080/22221751.202 0.1723441 PMID: 32005086

5. Li Q, Guan X, Wu P, Wang X, Zhou L, Tong Y, et al. Early Transmission Dynamics in Wuhan, China, of Novel CoronavirusInfected Pneumonia. N Engl J Med. 2020;382(13):1199-207. https://doi.org/10.1056/NEJMoa2001316 PMID: 31995857

6. Coronavirus disease (COVID-19) outbreak Geneva: World Health Organization; 2020. [Accessed 3 May 2020]. Available from: https://www.who.int/emergencies/diseases/ novel-coronavirus-2019

7. Li F. Structure, Function, and Evolution of Coronavirus Spike Proteins. Annu Rev Virol. 2016;3(1):237-61. https://doi. org/10.1146/annurev-virology-110615-042301 PMID: 27578435

8. Chan JF, Kok KH, Zhu Z, Chu H, To KK, Yuan S, et al. Genomic characterization of the 2019 novel human-pathogenic coronavirus isolated from a patient with atypical pneumonia after visiting Wuhan. Emerg Microbes Infect. 2020;9(1):22136. https://doi.org/10.1080/22221751.2020.1719902 PMID: 31987001

9. Lu R, Zhao X, Li J, Niu P, Yang B, Wu H, et al. Genomic characterisation and epidemiology of 2019 novel coronavirus: implications for virus origins and receptor binding. Lancet. 2020;395(10224):565-74. https://doi.org/10.1016/S01406736(20)30251-8 PMID: 32007145

10. Ceraolo C, Giorgi FM. Genomic variance of the 2019-nCoV coronavirus. J Med Virol. 2020;92(5):522-8. https://doi. org/10.1002/jmv.25700 PMID: 32027036

11. Lau SK, Woo PC, Li KS, Huang Y, Tsoi HW, Wong BH, et al. Severe acute respiratory syndrome coronaviruslike virus in Chinese horseshoe bats. Proc Natl Acad Sci USA. 2005;102(39):14040-5. https://doi.org/10.1073/ pnas.0506735102 PMID: 16169905

12. Ge XY, Li JL, Yang XL, Chmura AA, Zhu G, Epstein JH, et al. Isolation and characterization of a bat SARS-like coronavirus that uses the ACE2 receptor. Nature. 2013;503(7477):535-8. https://doi.org/10.1038/nature12711 PMID: 24172901

13. Hu B, Zeng LP, Yang XL, Ge XY, Zhang W, Li B, et al. Discovery of a rich gene pool of bat SARS-related coronaviruses provides new insights into the origin of SARS coronavirus. PLoS Pathog. 2017;13(11):e1006698. https://doi.org/10.1371/journal. ppat.1006698 PMID: 29190287

14. Yang XL, Hu B, Wang B, Wang MN, Zhang Q, Zhang W, et al. Isolation and Characterization of a Novel Bat Coronavirus Closely Related to the Direct Progenitor of Severe Acute Respiratory Syndrome Coronavirus. J Virol. 2015;90(6):3253-6. https://doi.org/10.1128/JVI.02582-15 PMID: 26719272

15. Graham RL, Baric RS. Recombination, reservoirs, and the modular spike: mechanisms of coronavirus cross-species transmission. J Virol. 2010;84(7):3134-46. https://doi. org/10.1128/JVI.01394-09 PMID: 19906932

16. Li W, Moore MJ, Vasilieva N, Sui J, Wong SK, Berne MA, et al. Angiotensin-converting enzyme 2 is a functional receptor for the SARS coronavirus. Nature. 2003;426(6965):450-4. https:// doi.org/10.1038/nature02145 PMID: 14647384

17. Wan Y, Shang J, Graham R, Baric RS, Li F. Receptor recognition by novel coronavirus from Wuhan: An analysis based on decade-long structural studies of SARS coronavirus. J Virol. 2020;94(7):eo0127-20. https://doi.org/10.1128/JVI.00127-20 PMID: 31996437

18. Letko M, Marzi A, Munster V. Functional assessment of cell entry and receptor usage for SARS-CoV-2 and other lineage B betacoronaviruses. Nat Microbiol. 2020;5(4):562-9. https:// doi.org/10.1038/s41564-020-0688-y PMID: 32094589

19. Keng CT, Zhang A, Shen S, Lip KM, Fielding BC, Tan TH, et al. Amino acids 1055 to 1192 in the $S_{2}$ region of severe acute respiratory syndrome coronavirus $S$ protein induce neutralizing antibodies: implications for the development of vaccines and antiviral agents. J Virol. 2005;79(6):3289-96. https://doi. org/10.1128/JVI.79.6.3289-3296.2005 PMID: 15731223

20. Lip KM, Shen S, Yang X, Keng CT, Zhang A, Oh HL, et al. Monoclonal antibodies targeting the HR2 domain and the region immediately upstream of the $\mathrm{HR}_{2}$ of the $\mathrm{S}$ protein neutralize in vitro infection of severe acute respiratory syndrome coronavirus. J Virol. 2006;80(2):941-50. https://doi. org/10.1128/JVI.80.2.941-950.2006 PMID: 16378996

21. Ng OW, Keng CT, Leung CS, Peiris JS, Poon LL, Tan YJ. Substitution at aspartic acid 1128 in the SARS coronavirus spike glycoprotein mediates escape from a S2 domaintargeting neutralizing monoclonal antibody. PLoS One. 2014;9(7):e102415. https://doi.org/10.1371/journal. pone.0102415 PMID: 25019613

22. ter Meulen J, van den Brink EN, Poon LL, Marissen WE, Leung CS, Cox F, et al. Human monoclonal antibody combination against SARS coronavirus: synergy and coverage of escape mutants. PLoS Med. 2006;3(7):e237. https://doi.org/10.1371/ journal.pmed.0030237 PMID: 16796401

23. Zheng Z, Teo SHC, Arularasu SC, Liu Z, Mohd-Ismail NK, Mok CK, et al. Contribution of Fc-dependent cell-mediated activity of a vestigial esterase-targeting antibody against $\mathrm{H}_{5} \mathrm{~N} 6$ virus infection. Emerg Microbes Infect. 2020;9(1):95-110. https:// doi.org/10.1080/22221751.2019.1708215 PMID: 31906790

24. Nakamura T, Yamada KD, Tomii K, Katoh K. Parallelization of MAFFT for large-scale multiple sequence alignments. Bioinformatics. 2018;34(14):2490-2. https://doi.org/10.1093/ bioinformatics/bty121 PMID: 29506019

25. Waterhouse AM, Procter JB, Martin DM, Clamp M, Barton GJ. Jalview Version 2--a multiple sequence alignment editor and analysis workbench. Bioinformatics. 2009;25(9):118991. https://doi.org/10.1093/bioinformatics/btpo33 PMID: 19151095

26. Kumar S, Stecher G, Li M, Knyaz C, Tamura K. MEGA X: Molecular Evolutionary Genetics Analysis across Computing Platforms. Mol Biol Evol. 2018;35(6):1547-9. https://doi. org/10.1093/molbev/msyo96 PMID: 29722887

27. Elbe S, Buckland-Merrett G. Data, disease and diplomacy: GISAID's innovative contribution to global health. Glob Chall. 2017;1(1):33-46. https://doi.org/10.1002/gch2.1018 PMID: 31565258

28. Tian X, Li C, Huang A, Xia S, Lu S, Shi Z, et al. Potent binding of 2019 novel coronavirus spike protein by a SARS coronavirusspecific human monoclonal antibody. Emerg Microbes Infect. 2020;9(1):382-5. https://doi.org/10.1080/22221751.2020.1729 o69 PMID: 32065055

29. Wrapp D, Wang N, Corbett KS, Goldsmith JA, Hsieh CL, Abiona 0 , et al. Cryo-EM structure of the 2019-nCoV spike in the prefusion conformation. Science. 2020;367(6483):1260-3. https://doi.org/10.1126/science.abb2507 PMID: 32075877

30. Poh WP, Narasaraju T, Pereira NA, Zhong F, Phoon MC, Macary $\mathrm{PA}$, et al. Characterization of cytotoxic T-lymphocyte epitopes and immune responses to SARS coronavirus spike DNA vaccine 
expressing the RGD-integrin-binding motif. J Med Virol. 2009;81(7):1131-9. https://doi.org/10.1002/jmv.21571 PMID: 19475608

31. Miyoshi-Akiyama T, Ishida I, Fukushi M, Yamaguchi K,

Matsuoka Y, Ishihara T, et al. Fully human monoclonal antibody directed to proteolytic cleavage site in severe acute respiratory syndrome (SARS) coronavirus S protein neutralizes the virus in a rhesus macaque SARS model. J Infect Dis. 2011;203(11):1574-

81. https://doi.org/10.1093/infdis/jiro84 PMID: 21592986

32. Zhong X, Yang H, Guo ZF, Sin WY, Chen W, Xu J, et al. B-cell responses in patients who have recovered from severe acute respiratory syndrome target a dominant site in the $\mathrm{S}_{2}$ domain of the surface spike glycoprotein. J Virol. 2005;79(6):34018. https://doi.org/10.1128/JVI.79.6.3401-3408.2005 PMID: 15731234

33. Duan J, Yan X, Guo X, Cao W, Han W, Qi C, et al. A human SARS-CoV neutralizing antibody against epitope on S2 protein. Biochem Biophys Res Commun. 2005;333(1):186-93. https:// doi.org/10.1016/j.bbrc.2005.05.089 PMID: 15939399

\section{License, supplementary material and copyright}

This is an open-access article distributed under the terms of the Creative Commons Attribution (CC BY 4.0) Licence. You may share and adapt the material, but must give appropriate credit to the source, provide a link to the licence and indicate if changes were made.

Any supplementary material referenced in the article can be found in the online version.

This article is copyright of the authors or their affiliated institutions, 2020. 